\title{
BMJ Open Oral health assessment of older adults living in long-term care facilities by non-dental professionals: a systematic review protocol of instruments
}

\author{
Lorrany Gabriela Rodrigues (D) ," Mario Vianna Vettore, ${ }^{1}$ \\ Isadora Lemos Figueiredo, ${ }^{1}$ Aline Araújo Sampaio, ${ }^{2}$ Raquel Conceição Ferreira (I) ${ }^{1}$
}

To cite: Rodrigues LG, Vettore MV, Figueiredo IL, et al. Oral health assessment of older adults living in long-term care facilities by non-dental professionals: a systematic review protocol of instruments. BMJ Open 2021;11:e050289. doi:10.1136/ bmjopen-2021-050289

- Prepublication history and additional supplemental material for this paper are available online. To view these files, please visit the journal online. (http://dx.doi.org/10.1136/ bmjopen-2021-050289).

Received 16 February 2021 Accepted 09 August 2021

Check for updates

(c) Author(s) (or their employer(s)) 2021. Re-use permitted under CC BY-NC. No commercial re-use. See rights and permissions. Published by BMJ.

${ }^{1}$ Department of Social and Preventive Dentistry, Federal University of Minas Gerais, Belo Horizonte, Brazil

${ }^{2}$ Department of Dental Clinical, Pathology and Surgery, Federal University of Minas Gerais, Belo Horizonte, Brazil

Correspondence to

Dr Raquel Conceição Ferreira;

raquelcf@ufmg.br

\section{ABSTRACT}

Introduction Regular oral health assessment among older adults living in long-term care facilities (LTCF) can improve their oral health. Different instruments have been developed and used to evaluate the oral health of institutionalised older people by non-dental professionals. These instruments must demonstrate adequate measurement properties. This systematic review aims to examine the studies describing the instruments employed to assess the oral health of older adults living in LTCF by non-dental professionals. The study will also evaluate the measurement properties of such instruments using the checklist proposed by the Consensus-based Standards to select health Measurement Instruments (COSMIN). Methods and analysis Studies describing the development of instruments for assessing oral health of institutionalised older adults by non-dental professionals will be included. Studies assessing at least one measurement property (validity, reliability or responsiveness) will be also considered. Electronic searches will be conducted on MEDLINE (PubMed, Ovid), Embase, Web of Science, Scopus and LILACS databases. Two independent reviewers will select the studies and will extract data concerning the characteristics of the research and the instrument. The measurement properties will be evaluated using the COSMIN checklist. The Grading of Recommendations, Assessment, Development and Evaluation approach will be used to grade the quality (or certainty) of evidence and strength of recommendations. Ethics and dissemination No ethical approval is required. The results will be submitted for publication to a peer-review journal and presented at relevant conferences. PROSPERO registration number CRD42020191479.

\section{INTRODUCTION}

Population ageing is a worldwide process resulting in a burden of chronic agerelated conditions that constitute a significant challenge for health systems. ${ }^{1}$ Since frailty and functional dependency increase with ageing, it can be expected an increase of institutionalisation among older people. ${ }^{2}$ Institutionalised older adults present greater dependency to
Strengths and limitations of this study

- This study will be an evidence for implementing best oral healthcare practices in long-term care facilities.

- The systematic review will be guided by validated and consolidated instruments and tools. The assessment of the risk of bias and the quality of the evidence will be carried out using Consensus-based Standards to select health Measurement Instruments checklist, and Grading of Recommendations, Assessment, Development and Evaluation approach and will be reported according to the Preferred Reporting Items for Systematic Reviews and Meta-Analyses.

- The systematic review will allow us to support an implementation study for oral care.

- This review will be limited to studies published in English, Spanish and Portuguese languages.

perform routine activities on daily basis than non-institutionalised ones, including dependency to perform oral hygiene. ${ }^{3}$ The decline on manual dexterity and vision impairment may affect their ability to maintain an adequate level of oral hygiene and, cognitive and physical disabilities may have a negative impact on the motivation for oral health self-care. ${ }^{4}$ Over the recent years, the complex interrelationships between frailty, cognitive decline and poor self-care related to oral health in older adults have been drawn attention due to the increase of tooth retention in this age group. The latter has been a common finding in dental surveys in different countries. The longer tooth retention may be associated with a higher prevalence of oral diseases, such as tooth and root decay and periodontal disease. ${ }^{4}$ Older adults with missing teeth might also experience oral functional impairments due to poor adaptation of dental prostheses. Another problem is the paucity of public dental services for older 
adults in some places, where emergency dental care is the only service available ${ }^{4}$ and there is a shortage of oral health professionals working at long-term care facilities (LTCF).

The maintenance of oral health must be part of the healthcare in $\mathrm{LTCF}^{5}$, which in turn may prevent the occurrence of systemic diseases such as aspiration pneumonia. ${ }^{6}$ Regular oral examination can detect early dental problems, reducing oral health deterioration and the need of complex dental treatments. The systematic evaluation of oral health can also support the need for adoption, reinforcement or improvement of guidelines for oral health promotion and preventive strategies in institutionalised older adults. In this context, caregivers are responsible for healthcare of older people in the daily routine in LTCF. Thus, they have a crucial role in performing oral health evaluation as part of the healthcare plan. ${ }^{4578}$

Some instruments have been used to evaluate the oral health of institutionalised older people by non-dental professionals, such as Oral Health Assessment Tool, ${ }^{9}$ Minimal Data Set, ${ }^{10}$ Dental Hygiene Registration, ${ }^{11}$ Oral Health Screening Tool for Nursing Personnel ${ }^{12}$ and oral health-related section of the Resident Assessment Instrument. ${ }^{13}$ The use of appropriate instruments is paramount to identify oral health problems, favouring the daily oral care plan, and referral to a dental service, if necessary. ${ }^{7113-15}$ Although such instruments must demonstrate adequate measurement properties, there is evidence that some of them may present methodological shortcomings. ${ }^{15} 16$ Two previous systematic reviews have evaluated the instruments used for oral health assessment. ${ }^{15} 16$ However, both studies have not focused on institutionalised older adults. ${ }^{1516}$ Thus, an update seems timely and legitimate.

Implementing best practices in LTCF, such as oral health assessment, should be evidence based and adapted to each context. This review can support the identification of the most suitable instrument for oral health evaluation of institutionalised older adults. The results may also indicate the need for additional assessment of the measurement properties of the existing instruments or highlight the need to develop and test a new one. This systematic review aims to identify the studies that described instruments developed to assess the oral health of older adults in LTCF by non-dental professionals and to evaluate their measurement properties.

\section{METHODS AND ANALYSIS}

This study protocol has been prepared according to the 2015 Preferred Reporting Items for Systematic Reviews and Meta-Analyses (PRISMA) Protocols guidelines (online supplemental material 1). The systematic review also will be reported according to the PRISMA ${ }^{17}$ and Consensus-based Standards to select health
Measurement Instruments (COSMIN) checklist for systematic reviews. ${ }^{18}$

\section{Review question}

What instruments have been used to assess the oral health of institutionalised older people by non-dental professionals?

Do these instruments present acceptable measurement properties?

\section{Patient and public involvement}

No patients were involved in the design of the study.

\section{Inclusion criteria}

- The instrument must aim to assess oral health of institutionalised older adults.

- The instrument must be applied by non-dental professionals, such as caregivers and nurses.

- Studies must describe the development or the assessment of at least one measurement property of the instrument (validity, reliability or responsiveness).

- Studies published in English, Spanish or Portuguese.

- No publication date or publication status restrictions will be imposed.

\section{Types of studies}

This review will include epidemiological studies, including validation studies and observational studies reporting measurement properties of the instruments used to assess oral health of institutionalised older adults by non-dental professionals.

\section{Search strategy}

The studies will be identified by searching four electronic databases: MEDLINE (PubMed), Embase, Web of Science, Scopus and LILACS (Latin American and Caribbean Literature in Health Sciences). Besides, a search on Google Scholar and OpenGray will identify further potential relevant articles. The reference lists of included articles will also be screened.

According to the COSMIN checklist for systematic reviews, the search strategy will follow up four key elements of the review aim: (1) Construct (related to Oral Health); (2) Population (related to Age, Long-term care and Caregivers); (3) Type of instrument (related to Assessment Tool) and (4) Measurement properties (using a PubMed search filter to find studies on measurement properties. ${ }^{19}$ The search blocks site will be used to assist the construction of the search strategy blocks (https://blocks.bmionline.nl/). The index terms were used to develop a full search strategy for MEDLINE (online supplemental material 2).

Screening and eligibility assessment will be performed independently by two trained reviewers. They will evaluate the relevant articles according to abstract and then the full text, respectively. Disagreements between the reviewers will be resolved by consensus. The number of identified articles, the screening and eligibility steps, and 


\section{Box 1 Data to be extracted from included studies}

Characteristics of the study
Aim of the study.
Study design.
Sample size.
Country of data collection.
Settings.
Measurement properties evaluated.
Limitations of the study.
Chanclusions.
Chacteristics of the instrument
Name of the instrument.
Professionals who applied the instrument.
Need for training to apply the instrument.
Instrument items.

the included studies will be shown using the flow diagram proposed by PRISMA.

\section{Data extraction}

Two reviewers will perform data extraction independently using a prepiloted spreadsheet developed for this purpose. A third reviewer will be consulted in case of disagreements. The characteristics of the study and the instrument that will be extracted from each article are presented in box 1 .

The extracted data from all included studies will be qualitatively synthesised and presented in tables along with a narrative summary.

\section{Assessment of methodological quality}

Two trained reviewers will independently evaluate the quality of the included studies using the COSMIN Risk of Bias checklist. ${ }^{20}$ The COSMIN aims to improve the selection of patient-reported outcome measures (PROMs) instruments in research and clinical practice through tools to select the most appropriate instrument. Despite focusing on PROMs, it can also be adapted for other purposes, such as instruments for measuring clinical outcomes. ${ }^{18}$ According to COSMIN, three domains must be evaluated to assess the quality of the instrument: reliability, validity and responsiveness. Each domain contains one or more measurement properties (table 1$).{ }^{18}$ Figure 1

\begin{tabular}{ll}
\hline Table 1 & Definitions of measurement properties \\
\hline Domain & Definition \\
\hline Reliability & $\begin{array}{l}\text { The degree to which the measurement is } \\
\text { free from measurement error }\end{array}$ \\
Validity & $\begin{array}{l}\text { The degree to which a PROM measures } \\
\text { the construct(s) it purports to measure }\end{array}$ \\
Responsiveness & $\begin{array}{l}\text { The ability of a PROM to detect change } \\
\text { over time in the construct to be measured }\end{array}$ \\
\hline
\end{tabular}

PROM, patient-reported outcome measure. presents the steps to assess the methodological quality and grading the quality of evidence.

The COSMIN Risk of Bias checklist will be used to assess the methodological quality of single studies included in the systematic review. The checklist contains standards referring to design requirements and preferred statistical methods of studies on measurement properties, containing 10 boxes: with one standard for PROM development and nine for measurement properties: content validity, structural validity, internal consistency, cross-cultural validity/measurement invariance, reliability, measurement error, criterion validity, hypotheses testing for construct validity and responsiveness. A standard COSMIN Excel spreadsheet will be used for each box. The measurement properties evaluated in a study will determine which boxes should be completed. A fourpoint rating scale will be assigned for each study as follows 'very good', 'adequate', 'doubtful' or 'inadequate'. The overall rating of the quality of each study will be determined by taking the lowest rating of any standard in the box. ${ }^{18}$ Next, the results of each study on a measurement property will be rated against the updated criteria for good measurement properties. Each result will be rated as either sufficient $(+)$, insufficient $(-)$ or indeterminate (?). ${ }^{18}$ The overview tables will be used to assess whether the results of two or more articles using the same instrument are consistent with each other to obtain the pooled result against criteria of good measurement properties.

\section{Grading the quality of evidence}

After pooling all evidence per measurement property per instrument and rating the pooled result against the criteria for good measurement properties, the quality of the evidence will be graded based on a modified Grading of Recommendations, Assessment, Development and Evaluation (GRADE) approach for systematic reviews. The quality of the evidence refers to the confidence that the summarised result is valid. The GRADE approach classifies the certainty in the findings as high, moderate, low or very low. Four of the five factors of the GRADE approach will be used for evaluating measurement properties: risk of bias (the methodological quality of the studies), inconsistency (unexplained inconsistency of results across studies), imprecision (total sample size of available studies) and indirectness (evidence from a different population than the population of interest in the review). ${ }^{21}$ The quality of the evidence will be performed by two trained reviewers independently. The GRADE approach will be used to downgrade evidence when there are concerns about the quality of evidence. The starting point is always the assumption that the obtained pooled result if of 'high' quality level. The quality of evidence can be subsequently downgraded by one or two levels per factor to moderate, low or very low evidence if there is imprecision (low sample size) or serious or very serious risk of bias, inconsistency, indirectness. The quality of evidence can even be downgraded by three levels when 


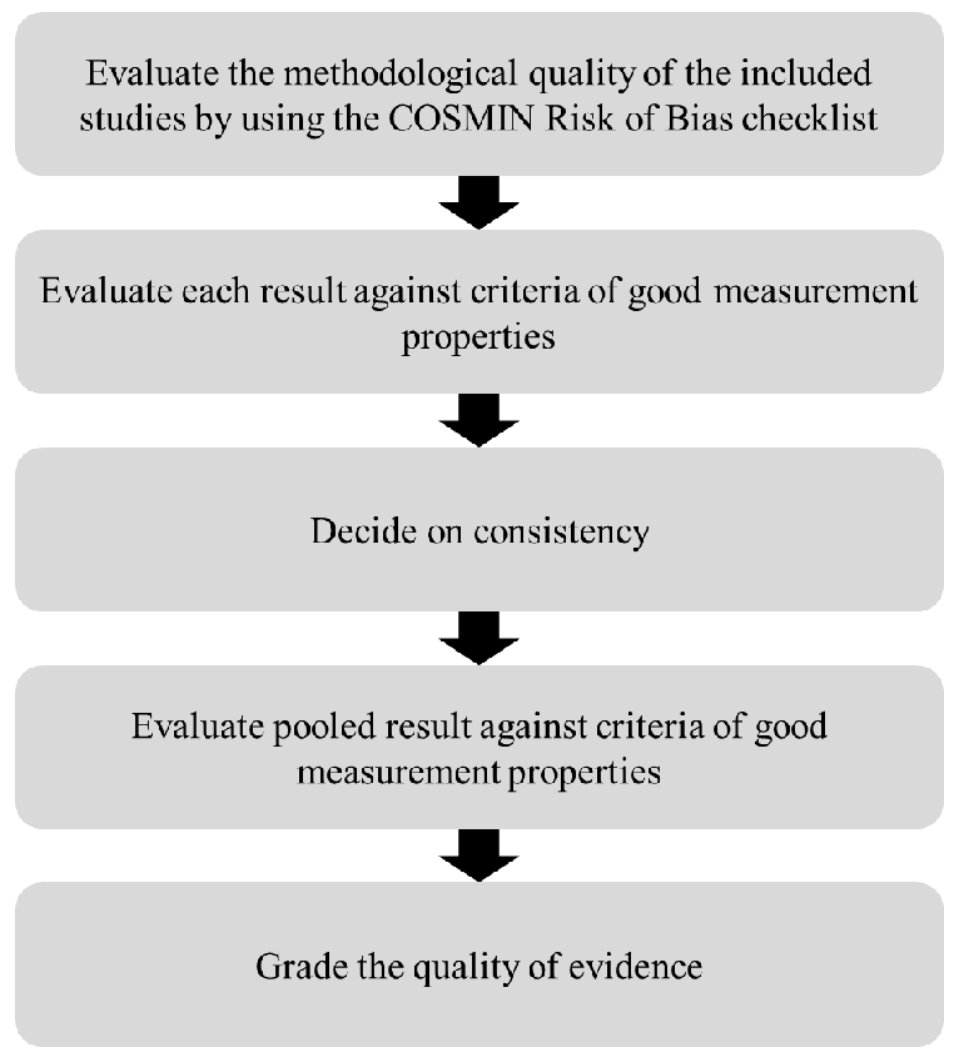

Figure 1 Flow chart of methodological quality evaluation and grading the quality of evidence source: adapted from Mokkink et $a l^{18}$. COSMIN, Consensus-based Standards to select health Measurement Instruments.

the evidence is based on only one inadequate study (extremely serious risk of bias).

This review also aims to assess the interpretability, which corresponds to the qualitative meaning for the quantitative scores obtained by applying the instrument, and the feasibility, that is, the ease of use of the instruments. ${ }^{18}$ Finally, based on the evaluations performed, this study will might be able to suggest recommendations on the most suitable instrument for non-dental professionals assessing the oral health of institutionalised older adults.

\section{Ethics and dissemination}

No ethical approval is required. The results will be submitted for publication to a scientific journal and presented at relevant conferences.

Contributors LGR and RCF conceived the study. LGR and ILF drafted the manuscript. AAS, MVV and RCF revised the manuscript. All authors approved the final version to be published. LGR is the guarantor of the review.

Funding RCF received financial support from FAPEMIG, Brazil (Fundação de Amparo à Pesquisa do Estado de Minas Gerais-Programa Pesquisador MineiroPPM-00603-18). This study is part of master degree dissertation developed in Universidade Federal de Minas Gerais (School of Dentistry). This study was financed in part by the Coordenação de Aperfeiçoamento de Pessoal de Nível SuperiorBrasil (CAPES)-Finance Code 001.

Disclaimer The sponsors have not been involved in the study design; in the writing of this protocol; and in the decision to submit the paper for publication.

Competing interests None declared.

Patient consent for publication Not required.

Provenance and peer review Not commissioned; externally peer reviewed.
Supplemental material This content has been supplied by the author(s). It has not been vetted by BMJ Publishing Group Limited (BMJ) and may not have been peer-reviewed. Any opinions or recommendations discussed are solely those of the author(s) and are not endorsed by BMJ. BMJ disclaims all liability and responsibility arising from any reliance placed on the content. Where the content includes any translated material, BMJ does not warrant the accuracy and reliability of the translations (including but not limited to local regulations, clinical guidelines, terminology, drug names and drug dosages), and is not responsible for any error and/or omissions arising from translation and adaptation or otherwise.

Open access This is an open access article distributed in accordance with the Creative Commons Attribution Non Commercial (CC BY-NC 4.0) license, which permits others to distribute, remix, adapt, build upon this work non-commercially, and license their derivative works on different terms, provided the original work is properly cited, appropriate credit is given, any changes made indicated, and the use is non-commercial. See: http://creativecommons.org/licenses/by-nc/4.0/.

\section{ORCID iDs}

Lorrany Gabriela Rodrigues http://orcid.org/0000-0002-9531-5392

Raquel Conceição Ferreira http://orcid.org/0000-0001-8897-9345

\section{REFERENCES}

1 WHO. World health statistics 2020, 2020. Available: https://apps. who.int/iris/bitstream/handle/10665/332070/9789240005105-eng.pdf

2 Hajek A, Brettschneider C, Lange C, et al. Longitudinal predictors of institutionalization in old age. PLoS One 2015;10:e0144203.

3 Farias IPSE, Sousa SAde, Almeida LdeFDde, et al. Does noninstitutionalized elders have a better oral health status compared to institutionalized ones? A systematic review and meta-analysis. Cien Saude Colet 2020;25:2177-92.

4 Razak PA, Richard KMJ, Thankachan RP, et al. Geriatric oral health: a review article. J Int Oral Health 2014;6:110-6.

5 Klotz A-L, Zajac M, Ehret J, et al. Short-term effects of a deterioration of general health on the oral health of nursing-home residents. Clin Interv Aging 2020;15:29-38. 
6 Sjögren P, Nilsson E, Forsell M, et al. A systematic review of the preventive effect of oral hygiene on pneumonia and respiratory tract infection in elderly people in hospitals and nursing homes: effect estimates and methodological quality of randomized controlled trials. J Am Geriatr Soc 2008;56:2124-30.

7 Wårdh I, Jonsson M, Wikström M. Attitudes to and knowledge about oral health care among nursing home personnel-an area in need of improvement. Gerodontology 2012;29:e787-92.

8 Lindmark U, Jansson $\mathrm{H}$, Lannering $\mathrm{C}$, et al. Oral health matters for the nutritional status of older persons-A population-based study. $J$ Clin Nurs 2018;27:1143-52.

9 Chalmers JM, King PL, Spencer AJ, et al. The oral health assessment tool--validity and reliability. Aust Dent $J$ 2005;50:191-9.

10 Hawes C, Morris JN, Phillips CD, et al. Reliability estimates for the minimum data set for nursing home resident assessment and care screening (MDS). Gerontologist 1995;35:172-8.

11 Fjeld KG, Eide $\mathrm{H}$, Mowe M, et al. Dental hygiene registration: development, and reliability and validity testing of an assessment scale designed for nurses in institutions. J Clin Nurs 2017;26:1845-53

12 Tsukada S, Ito K, Stegaroiu R, et al. An oral health and function screening tool for nursing personnel of long-term care facilities to identify the need for dentist referral without preliminary training. Gerodontology 2017;34:232-9.

13 Krausch-Hofmann S, De Almeida Mello J, Declerck D, et al. The oral health-related section of the interRAl: evaluation of test content validity by expert rating and assessment of potential reasons for inaccurate assessments based on focus group discussions with caregivers. Gerodontology 2019;36:382-94.

14 Kossioni AE, Hajto-Bryk J, Janssens B, et al. Practical guidelines for physicians in promoting oral health in frail older adults. J Am Med Dir Assoc 2018;19:1039-46.

15 Chalmers JM, Pearson A. A systematic review of oral health assessment by nurses and carers for residents with dementia in residential care facilities. Spec Care Dentist 2005;25:227-33.

16 Everaars B, Weening-Verbree LF, Jerković-Ćosić K, et al. Measurement properties of oral health assessments for non-dental healthcare professionals in older people: a systematic review. BMC Geriatr 2020;20:1-18.

17 Moher D, Liberati A, Tetzlaff J, et al. Preferred reporting items for systematic reviews and meta-analyses: the PRISMA statement. BMJ 2009;339:b2535.

18 Mokkink C, Prinsen AC, Patrick J. COSMIN methodology for systematic reviews of patient - reported outcome measures (PROMs). Amsterdam: Public Health Reasearch Institute, 2018: 1-78.

19 Liao L, Xiao LD, Chen $\mathrm{H}$, et al. Nursing home staff experiences of implementing mentorship programmes: a systematic review and qualitative meta-synthesis. J Nurs Manag 2020;28:188-98.

20 Mokkink LB, de Vet HCW, Prinsen CAC, et al. COSMIN risk of bias checklist for systematic reviews of patient-reported outcome measures. Qual Life Res 2018;27:1171-9.

21 Schünemann HJ, Brozek J, Guyatt G, et al. GRADE Handbook Handbook for grading the quality of evidence and the strength of recommendations using the GRADE approach, 2013. Available: https://gdt.gradepro.org/app/handbook/handbook.html 